\title{
Particle Control in the Sustained Spheromak Physics Experiment
}

R.D. Wood, D.N. Hill, E.B. Hooper, D. Buchenauer, H. McLean, Z. Wang, S. Woodruff, and G. Wurden

This article was submitted to $14^{\text {th }}$ International Conference on Plasma Surface Interactions, Rosenheim, Germany, May 22-26, 2000

\section{May 2000}

Lawrence

Livermore

National

Laboratory 


\section{DISCLAIMER}

This document was prepared as an account of work sponsored by an agency of the United States Government. Neither the United States Government nor the University of California nor any of their employees, makes any warranty, express or implied, or assumes any legal liability or responsibility for the accuracy, completeness, or usefulness of any information, apparatus, product, or process disclosed, or represents that its use would not infringe privately owned rights. Reference herein to any specific commercial product, process, or service by trade name, trademark, manufacturer, or otherwise, does not necessarily constitute or imply its endorsement, recommendation, or favoring by the United States Government or the University of California. The views and opinions of authors expressed herein do not necessarily state or reflect those of the United States Government or the University of California, and shall not be used for advertising or product endorsement purposes.

This is a preprint of a paper intended for publication in a journal or proceedings. Since changes may be made before publication, this preprint is made available with the understanding that it will not be cited or reproduced without the permission of the author.

This report has been reproduced

directly from the best available copy.

Available to DOE and DOE contractors from the

Office of Scientific and Technical Information

P.O. Box 62, Oak Ridge, TN 37831

Prices available from (423) 576-8401

http: / / apollo.osti.gov/ bridge/

Available to the public from the

National Technical Information Service

U.S. Department of Commerce

5285 Port Royal Rd., Springfield, VA 22161

http:/ / www.ntis.gov/

OR

Lawrence Livermore National Laboratory

Technical Information Department's Digital Library

http: / / www.llnl.gov/tid/Library.html 


\section{Particle Control in the Sustained Spheromak Physics Experiment}

R.D. Wood, D.N. Hill, E.B. Hooper, D. Buchenauer ${ }^{\mathrm{a}}$, H. McLean, Z. Wang ${ }^{\mathrm{b}}$, S. Woodruff, and G. Wurden ${ }^{\mathrm{b}}$

Lawrence Livermore National Laboratory, Livermore, CA

${ }^{a}$ Sandia National Laboratories, Livermore, CA

${ }^{b}$ Los Alamos National Laboratory, Los Alamos, NM

In this paper we report on density and impurity measurements in the Sustained Spheromak Physics Experiment (SSPX) which has recently started operation. The SSPX spheromak plasma is sustained by coaxial helicity injection for a duration of $2 \mathrm{msec}$ with peak toroidal currents of up to 0.5MA. The plasma-facing components consist of tungsten-coated copper to minimize sputtering. The surfaces are conditioned by a combination of baking at $150^{\circ} \mathrm{C}$, glow discharge cleaning, Titanium gettering, and pulse-discharge cleaning with helium plasmas. In this way we can achieve density control so that the plasma density $\left(\sim 1-4 \times 10^{20} \mathrm{~m}^{-3}\right)$ matches the gas input. Low-density operation is presently limited by breakdown requirements, but we hope that new gas valves with supersonic nozzles will allow for a further reduction in density. We find that the conditioning reduces the impurity radiation to the point where it is no longer important to the energy balance, and long-lived spheromak plasmas are obtained (decay times of $1.5 \mathrm{msec}$ ). 


\section{Introduction}

In this paper we discuss power and particle control for the SSPX (Sustained Spheromak Physics Experiment) spheromak device. SSPX began operation in 1999 after it was constructed as part of a renewed US program in alternate confinement concepts. In spheromaks, a very low aspect ratio (A 1.1) toroidal confinement geometry is produced by currents in the plasma itself (the plasma dynamo), rather than by external coils which necessarily thread the vacuum vessel. Elimination of the linked coils could lead to smaller, cheaper power plants. Furthermore, DC or AC potentials applied to external electrodes can sustain the spheromak plasma. At present, it is unknown if the spheromak configuration can provide sufficient energy confinement to allow the plasma to be heated to thermonuclear temperatures $(10 \mathrm{keV})$. Recent analysis of previous experimental data[1,2] suggested that adequate core energy confinement could be obtained in these devices and that performance might scale favorably to power reactors. The SSPX device was built to explore this question.

The spheromak plasma in SSPX is confined within an $\mathrm{R}=1.0 \mathrm{~m}, \mathrm{~h}=0.5 \mathrm{~m}, 1.2 \mathrm{~cm}$ thick copper flux conserver which serves to maintain the plasma shape via image currents flowing in it. A cross section of the device appears in Fig. 1; magnetic flux surfaces for an ideal MHD equilibrium computed with the CORSICA code are included. The confined plasma $(R=0.31 \mathrm{~m}, \mathrm{a}>0.25 \mathrm{~m}$ is isolated from the flux conserver by a thin (less than $1 \mathrm{~cm}$ wide at the midplane) scrape-off layer (SOL) plasma whose field lines encircle the plasma and connect to the electrode region at the top of the device. In the spheromak the magnetic field lines vary from almost completely poloidal (in the plane of the paper here) near the walls to completely toroidal at the magnetic axis at $\mathrm{R}=0.31 \mathrm{~m}$. The cross section of the shell was designed to minimize the volume of corner regions 
having open field lines, so it is everywhere conformal to the magnetic flux surfaces except for a $5 \mathrm{~cm}$ high toroidally uniform diagnostic slot encircling the midplane.

The spheromak plasma in SSPX is formed by coaxial injection: high voltage applied across the coaxial gap above the main flux conserver produces a radial discharge current. The current flowing down the central electrode produces a toroidal field in the gap and the resulting $\mathrm{j} \times \mathrm{B}$ force pushes the plasma down into the main chamber. $\mathrm{A}$ vacuum magnetic field directed radially across the gap, is pulled with the plasma and forms the poloidal field of the spheromak in the main chamber. In the final state, the plasma assumes a single-null divertor cross section as shown, with the x-point up in the injector region. Two capacitor banks supply the energy needed to form the spheromak: a $0.5 \mathrm{MJ} 10 \mathrm{kV}$ formation bank and a $1.5 \mathrm{MJ} 5 \mathrm{kV}$ sustainment bank.

In terms of plasma surface interactions and the scrape-off layer plasma, the main issues for the spheromak are as follows. Foremost is impurity generation by the high current discharge in the injector region. Peak surface-normal current density can reach $60 \mathrm{~A} / \mathrm{cm} 2$ and if the gas density is too low in this region, the current will be maintained by sputtering of the wall material. Secondly, we are concerned about sputtering from the walls of the flux conserver because the scrape-off layer plasma is relatively thin. To mitigate these effects we have coated the copper surfaces with a $100 \mu \mathrm{m}$ thick layer of plasma-sprayed tungsten. This layer is more porous than we would like (as much as $20 \%$ porosity and we now rely on titanium gettering to retain impurities trapped in this layer. The interested reader should see Buchenauer, et al. in these proceedings. Density control is another issue for present-day spheromak experiments because the short pulse duration precludes real-time feedback and because breakdown requirements in the 
injector impose a minimum gas injection rate to reach the minimum of the Paschen curve. Careful attention to gas valve design can help here.

In the remainder of this paper we summarize the basic features of typical SSPX spheromak plasmas (Sect. II), discuss particle balance and density control in Section III, and in Section IV show how improved surface conditioning has reduced impurity radiation and improved performance.

\section{Spheromak Formation Experiments}

Present-day spheromaks operate without feedback control of the main plasma parameters due to their high injector currents and short pulse duration. However, the global properties of the plasma can be adjusted by setting the voltage on the capacitor bank, external circuit impedance, initial magnetic flux in the injector, and the amount of gas injected. Once set, the resulting plasmas can be highly reproducible as long as steady wall conditions are maintained.

Spheromak plasmas have four distinct phases: breakdown, formation (or ejection), sustainment, and decay. These features are illustrated by data from SSPX in Figure 2. The breakdown phase, which occurs in the first few microseconds of the pulse, places significant limits on the operating space of the device since enough gas must be injected to obtain Paschen breakdown. In SSPX we increased the radius of the coaxial source by about a factor of three over previous experiments and also widened the radial gap in order to improve the drive efficiency[3]. As a result, significantly more gas input is required for breakdown than in other devices and higher volume-average densities are obtained. We have managed to reduce the required gas input about a factor of four by creating a Penning discharge configuration in the injector. We expect to obtain a further 
similar reduction by modifying the gas valve nozzles to produce a supersonic gas jet, which is directed across the gap, thereby meeting the Paschen condition locally in front of each valve.

Following breakdown, the plasma current rises sharply during the formation phase, which lasts until about $0.2 \mathrm{msec}$ for the plasma shown here. During this time, if the radial current is large enough, the plasma in the injector will accelerate out into the flux conserver to form a spheromak plasma. The threshold current depends on the radius of the injector, the width of the coaxial region, and the strength of the initial magnetic field[4]. For the plasma shown here, the threshold current is $200 \mathrm{kA}$, as evidenced by the sudden rise in poloidal magnetic field in the main volume when the current exceeds this value at $50 \mu$ sec. During this time the plasma density rises to $2.5 \times 10^{14} \mathrm{~cm}^{-3}$, which represents about $25 \%$ of total gas input before breakdown.

After the fields and currents buildup in the main spheromak volume, the discharge enters the sustainment phase. If no additional energy is supplied to the injector, then the spheromak disconnects from the injector and the current decays on a timescale consistent with resistive dissipation of the magnetic fields. Given additional energy input from the sustainment bank, as in the case shown here, the spheromak plasma can be sustained for longer periods and the fields can continue to build as long as the current remains above the threshold value (otherwise the additional current just flows within the injector region). For SSPX, the decay without additional energy input lasts about $1 \mathrm{msec}$, depending on the cleanliness of the discharge. Adding energy with the sustainment bank can extend the pulse to more than $2 \mathrm{msec}$ duration. When the radiative losses are low, the decay is very gradual and the discharge usually terminates 
abruptly due to MHD activity. In dirty plasmas the fields decay steadily at a uniform rate.

\section{Density Control}

The performance of spheromaks depends on the plasma density and impurity content through some of the same physics as the tokamak. However, there is a further complication in that currents in the plasma itself produce the confining fields, so we expect low temperature resistive plasmas to have shorter decay times, lower fields, and worse confinement than hotter plasmas. A key measure for the spheromak is the quantity I/N (equivalently $\mathrm{j} / \mathrm{n}$ ), which can be related to the ratio of ohmic heating input power to impurity radiation loss power. Various authors have shown [5] this to be equivalent physics to the Murakami limit for tokamak density. As long as $\mathrm{j} / \mathrm{n}$ is greater than about $10^{-14} \mathrm{~A}-\mathrm{m}$, the ohmic heating will exceed the impurity radiation loss and the electron temperature will be transport limited. The exact value depends on the impurity species, but not very strongly on the impurity concentration since ultimately, both the resistivity and radiative losses scale together with $Z_{\text {eff }}$. Unlike the tokamak, no disruptive density limit is observed in the spheromak; rather, steady state low temperature plasmas with $\mathrm{P}_{\text {rad }} \sim \mathrm{P}_{\text {ohmic }}$ are produced.

In SSPX, we fuel the plasma by either a static prefill or localized gas puffing in the coaxial injector region about $250 \mu$ sec before the high voltage is applied; the short delay between gas and voltage helps keep most of the gas up in the injector region. Using a prefill produces spheromaks in which the plasma density is comparable to or higher than the initial neutral gas density (about $4-6 \times 10^{20} \mathrm{~m}^{-3}$ line average density). We mainly use this technique to obtain breakdown for helium-plasma pulsed discharge cleaning. Fueling with a short gas pulse just before firing the capacitor banks produces better 
spheromak plasmas with lower density. The resulting plasma density doesn't depend strongly on the size of the gas puff and at best, represents only about $50 \%$ of it (assuming uniform plasma density in the flux conserver); this is consistent with data from other spheromaks[6].

The evolution of the spheromak density during a discharge depends on whether the current in the spheromak (as opposed to the injector current) is sustained or is decaying. It is typically highest in the beginning, just after the spheromak plasma has fully formed (at about $0.3 \mathrm{msec}$ in Fig. 2). Without a sustainment pulse, the density will decay rapidly down to a low-level plateau, which is maintained until the spheromak field collapses. The plateau density is most likely sustained by recycling on the flux conserver wall since it doesn't depend on the size of the initial gas puff, but does increase with the spheromak field strength (confinement). It is not likely that gas flux through the diagnostic slot plays a significant role either, since even with a high prefill pressure of $10^{-3}$ torr, the particle flux $\left(\sim 10^{22}\right.$ atoms $\left./ \mathrm{sec}\right)$ is about an order of magnitude less than loss rate obtained using the $0.5 \mathrm{msec}$ density decay rate at the end of the pulse $\left(\mathrm{N} / \tau_{\mathrm{p}}>10^{23} / \mathrm{sec}\right)$

In sustained spheromaks the density depends strongly on whether the sustaining current is above the spheromak formation threshold, expressed as $\lambda=I / \psi$, where $\mathrm{I}$ is the current and $\psi$ is initial vacuum magnetic flux. If below, then there is only a weak dependence on $\lambda$ since most of the current and plasma remain in the injector region. As the current rises above the threshold, the injector plasma is swept out into the main chamber so that the spheromak density rises quickly and can be maintained at a high level. This behavior is evident from the data of Fig. 3, which shows the density, current, and spheromak poloidal field for two discharges, which differ only by the initial 
magnetic flux. For pulse 3407 the flux $\mathrm{p}$ has been lowered so that the current remains above the 100kA threshold value, while for 3410 the threshold current has been raised to near 190kA, which is higher than the discharge current. In this case, the density decays away rapidly to values similar to those for decaying spheromaks. Interestingly, this current maintains the poloidal field. A similar threshold current for maintaining spheromak density was observed in the CTX spheromak device[4].

Global particle balance studies show that less than $1 \%$ of the hydrogen fueling gas is retained in the tungsten walls after a plasma pulse. However, due to the porous nature of the tungsten surface, it takes many minutes for the hydrogen to be pumped away since it diffuses out at a rate proportional to $1 / \sqrt{ }$ t. Also, the inventory on the walls leads to a higher recycling coefficient. Therefore, we have used titanium gettering to trap the hydrogen more effectively and reduce the recycling. With gettering, we have lowered the density during the decay phase by almost an order of magnitude so

that the value of $\mathrm{j} / \mathrm{n}$ rose from $10^{-15} \mathrm{~A}-\mathrm{m}$ to above $10^{-14} \mathrm{~A}-\mathrm{m}$. We haven't yet quantified the improvement for sustained plasmas, though $\mathrm{j} / \mathrm{n}$ is well above $10^{-14} \mathrm{~A}-\mathrm{m}$. With gettering, the impurity radiation has dropped significantly, as discussed in the following Section.

\section{Impurity Control and Wall Conditioning}

Impurity control is very important for the spheromak, since it is thought that the core energy confinement depends on the electron temperature through magnetic turbulence so that a highly radiative cold plasma may have poor energy confinement and thus cannot be heated. In order to reduce the impurity concentration we coated the copper flux conserver a $100 \mu \mathrm{m}$ thick layer of plasma sprayed tungsten to minimize sputtering. It turns out, however, that these layers can be porous[7] and can absorb 
high levels of water. Surface analysis[8] shows concentrations of oxygen and carbon typical of metal surfaces with a measured oxide layer thickness at the surface of $15 \mathrm{~nm}$. Heating of the tungsten during plasma discharges would lead to a slow release of oxygen to the surface of the tungsten.

Initial conditioning consisted of baking to $150^{\circ} \mathrm{C}$ to remove water and hydrogen, followed by glow discharge cleaning (GDC) to remove surface hydrocarbons and oxides. Prior to bake out, water was the dominant gas in the vacuum chamber representing $\sim 80 \%$ of the total pressure. During bake out, the partial pressure of water increases an order of magnitude and after $\sim 100$ hours at $150^{\circ} \mathrm{C}$, the partial pressure decreases. After bake out and cooling (ambient) of the vessel, the partial pressure of water is an order of magnitude lower than before baking. Due to the limited pumping speed (500 liters/sec) and high backfill pressures (30 mTorr), the mass flow during GDC is small and therefore only moderate removal rates are attained. Even with baking and GDC the partial pressure of water can increase significantly after a spheromak discharge. During initial discharges on SSPX the partial pressure of water was as high as the partial pressure of the fueling gas, hydrogen. Other gases (methane, carbon monoxide and carbon dioxide) are also produced during these discharges. Water and volatile gas production during plasma discharges is attributed to the reduction of tungsten compounds (i. e., tungsten oxide) by hydrogen. Further details of the volatile gas production mechanisms and wall conditioning techniques are presented elsewhere[8].

Helium shot conditioning and titanium gettering further reduce impurities and lead to improve plasma performance. As measured with a residual gas analyzer (RGA), after 6-10 helium discharges, the partial pressure of water decreases an order of 
magnitude. After helium shot conditioning, the fueling gas is returned to hydrogen and a $10 \mathrm{~nm}$ thick coating of titanium (gettering) is evaporated onto the plasma facing surfaces of the flux conserver. The gettered surface pumps and buries water and the volatile gas species, thereby reducing the impurity levels in the plasma.

To characterize the impurity radiation, line emissions in the $100-1600 \AA$ spectral region are measured with an absolutely calibrated SPRED spectrograph[9, 10]. The spectrograph is mounted at the flux conserver midplane, has a tangential view of the magnetic axis and provides a time-integrated spectrum of the discharge. A pair of monochrometers having a similar view of the plasma as the SPRED provide, timeresolved line emissions in the 300-5500 $\AA$ spectral region. Time-resolved intensities of impurity emissions from the UV spectral region are obtained by cross-calibrating the monochrometers with the SPRED instrument. As shown in Fig. 4 (dashed curve), a typical spectrum of a discharge before helium shot conditioning and titanium gettering consists of low-Z impurities (carbon, nitrogen and oxygen). Tungsten and other metallic lines have not been observed, however, the amount of tungsten produced by sputtering during the various stages of the discharge is expected to be insignificant[11]. For the spectrum without gettering low charge states of oxygen, Li-like nitrogen and Li-like carbon are the dominant radiators. The solid curve in Fig. 4 shows a typical spectrum of a discharge after helium shot conditioning and with titanium gettering. Compared to the dashed curve, the Li-like CIV (1550 A) emission has decreased a factor of 10 and all of the lower charge states of carbon have burned through and are not radiating. Similarly, for nitrogen, the Li-like emission at $1240 \AA$ has dropped a factor of 5 and the lower charge states have burned through. For oxygen the Li-like OVI $(1032,1038 \AA)$ has increased a factor of three and the Be-Like (OV) lines at $630 \AA$ and $760 \AA$ have 
decreased; the lower charge states of oxygen have also burned through. The ratio of OVI to OV in the non-gettered discharge is $<1$, whereas in the case with gettering this ratio is 10 indicating a hotter, cleaner plasma.

Spectroscopic determination of the radiated power and an estimate of the temperature $\left(\mathrm{T}_{\mathrm{e}}\right)$ in the region of impurity emissions are obtained from the measured line brightness. Taking advantage of the strong temperature dependence of the excitation rates of Li-like transitions, measurements of the important Li-like emissions of carbon, nitrogen, and oxygen are used to provide an estimate of the electron temperature. The ratio of the measured brightness is directly proportional to the ratio of excitation rates. From the ratio of the measured brightness of the $2 s-2 p$ transition and the $2 s-3 p$ transition we estimate the electron temperature $\left(T_{e}\right)$ in the ungettered case to be on the order of $25 \mathrm{eV}$ and with gettering $\mathrm{T}_{\mathrm{e}} \sim 50-60 \mathrm{eV}$. The total radiated power in this spectral region is also determined from the measured brightness. For the ungettered discharge shown in Fig. 4 (dashed), assuming uniform emissivity over the plasma volume, the total radiated power determined spectroscopically is 140 MW. In the case with gettering the spectroscopically determined radiated power is $\sim 50 \mathrm{MW}$. The efficiency of transferring gun power to ohmic power is the $\lambda$-ratio of the spheromak to gun[3]. For these discharges the gun efficiency is $\sim 50 \%$ and the total gun power is 480 MW or $240 \mathrm{MW}$ of ohmic power. The spectroscopically determined radiated power represents $\sim 70 \%$ of the total ohmic input power in the ungettered case and $\sim 20 \%$ in the case with gettering.

The measured total radiated energy from a thermistor mounted at the midplane with a full horizontal cross-sectional view of the plasma shows radiated energy decreases with helium shot conditioning. Fig. 6 shows the fraction of radiated energy to 
injector input energy for an ensemble of discharges with gettering only and with gettering and helium shot conditioning. Discharges with helium shot conditioning radiate a factor of 4 less energy than discharges with only gettering. The balance of input energy goes into heating and mode analysis reveals increased MHD activity. Fig. 5 shows the time evolution of the edge poloidal field for a discharge with gettering and helium shot conditioning (solid curve) and one without (dashed). In the case with conditioning, the edge poloidal field decay time has increased a factor of 2 . The average electron temperature is proportional to the B-field energy decay time[12] and for the discharge with helium shot conditioning and gettering the calculated $T_{e} \geq 50 \mathrm{eV}$ compared to $\sim 25 \mathrm{eV}$ for discharges without conditioning.

\section{Summary}

In this paper we have considered density and impurity control for the SSPX spheromak. Presently, we are relying on a plasma-sprayed tungsten coating to reduce sputtering coupled with baking, hydrogen glow discharge cleaning and titanium gettering to reduce surface impurities and hydrogen recycling. With this combination we have been able to significantly reduce the concentration of carbon and nitrogen in the plasma and have lowered the density by more than a factor of two. In this way we are able to increase $\mathrm{j} / \mathrm{n}$ by more than an order of magnitude ( to $>10^{-14} \mathrm{~A}-\mathrm{m}$ ) and achieve burnout of most low-Z impurities. From the density and impurity behavior of the discharge, we conclude that the injector plasma is the main source of plasma ions and impurities, though most of the impurities are generated during the early formation phase. Further improvements in density control should be obtained with the installation of new gas valves which feature supersonic nozzles to increase the local gas density in the injector; in this way we can satisfy the requirements for Paschen 
breakdown with less total gas fueling. In addition, application of boron or lithium coatings is being studied to further decrease the oxygen content in the plasma.

\section{Acknowledgements}

We acknowledge helpful discussions with Cris Barnes of LANL and Tom Jarboe of the University of Washington. This work was performed under the auspices of US DOE by the University of California Lawrence Livermore National Laboratory under Contract No. W-7405-ENG-48 and DE-AC03-89ER51114.

\section{References}

1. T. K. Fowler, J. S. Hardwick, T. R. Jarboe, Comments Plasma Phys. Controll. Fus. 16 (1994).

2. E. B. Hooper, J. H. Hammer, C. W. Barnes et al., Fusion Techn. 29 (1996).

3. M. R. Brown, Martin, A., Fusion Technol. 30, 300 (1996).

4. C. W. Barnes, T. R. Jarboe, G. L. Marklin et al., Phys. Fluids B 2 (1990).

5. S. Ortolani, G. Rostagni, Nucl. Intrum. Methods 207, 353 (1983).

6. W. C. Turner, E. H. A. Granneman, C. W. Hartman et al., J. Appl. Phys. 52 (1981).

7. C. Garcia-Rosales, P. Franzen, H. Plank et al., J. Nucl. Mater 233-237, 803 (1996).

8. D. Buchenauer, B. E. Mills, R. D. Wood et al., These proceedings (2000).

9. B. C. Stratton, R. J. Fonk, K. Ida et al., Rev. Sci. Instrum. 57, 2043 (1986).

10. R. J. Fonk, A. T. Ramsey, R. V. Yelle, Appl. Optics 21, 2115 (1982).

11. E. B. Hooper, D. Ryutov, J. Nucl. Mater. 104-110, 278 (2000).

12. C. W. Barnes, T. R. Jarboe, I. Henins et al., Nucl. Fusion 24, 267 (1984). 


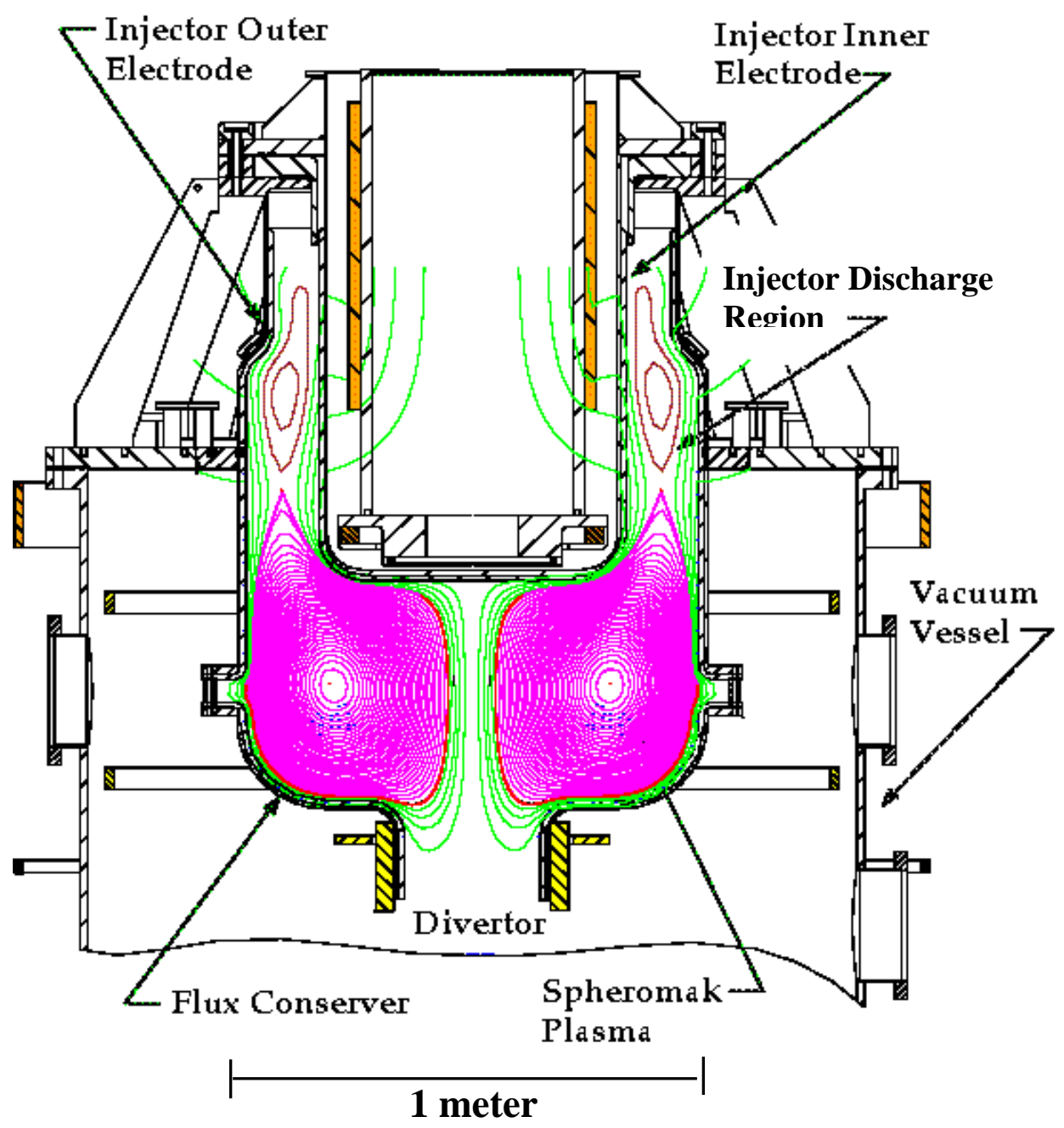

Figure 1. SSPX with Corsica equilibrium profile. 


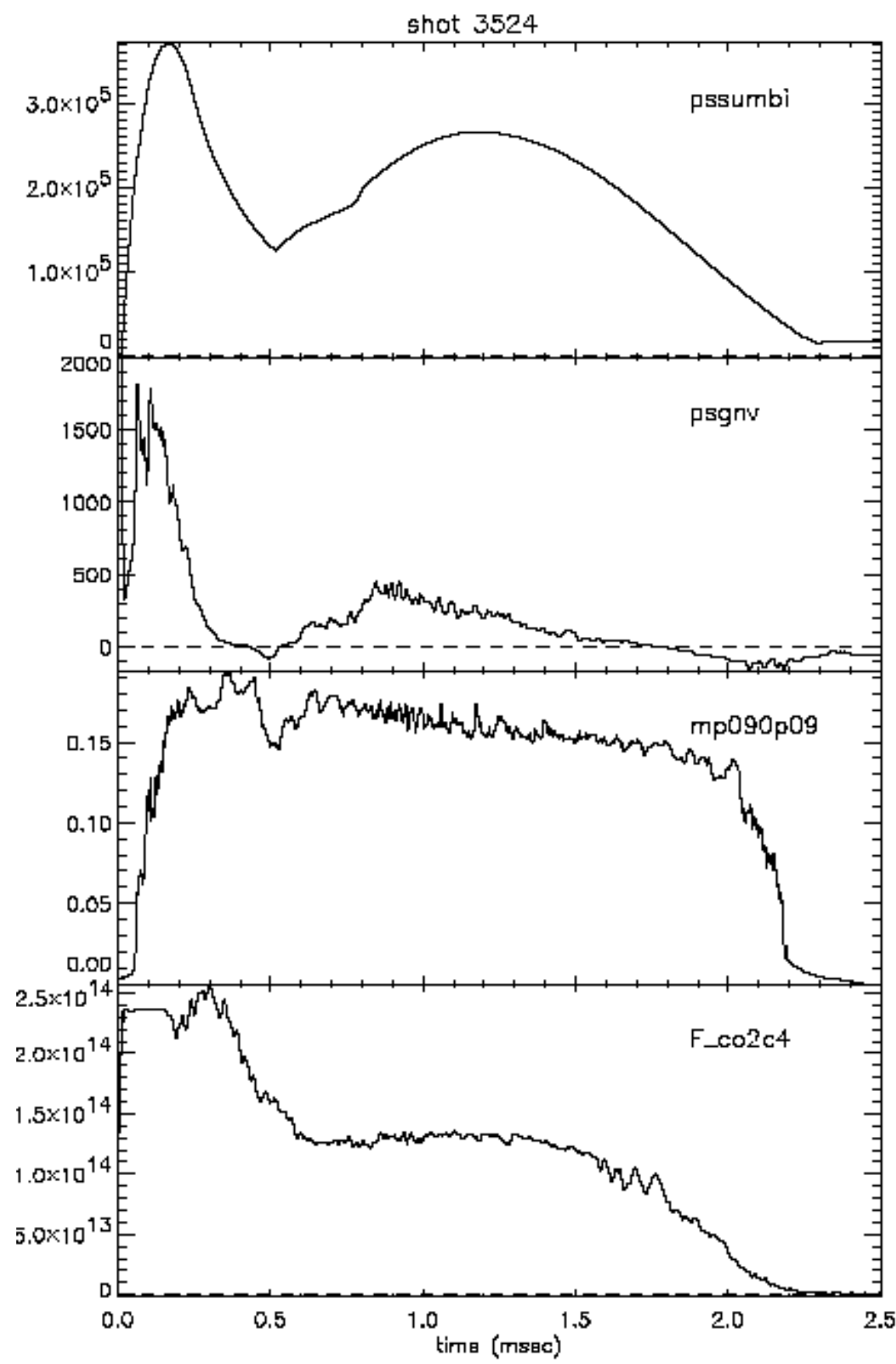

Figure 2. Typical sustained spheromak discharge. a) injector current $(A), b)$ injector voltage $(\mathrm{V}), \mathrm{c})$ midplane poloidal field $(\mathrm{T})$, and d) line average density. 


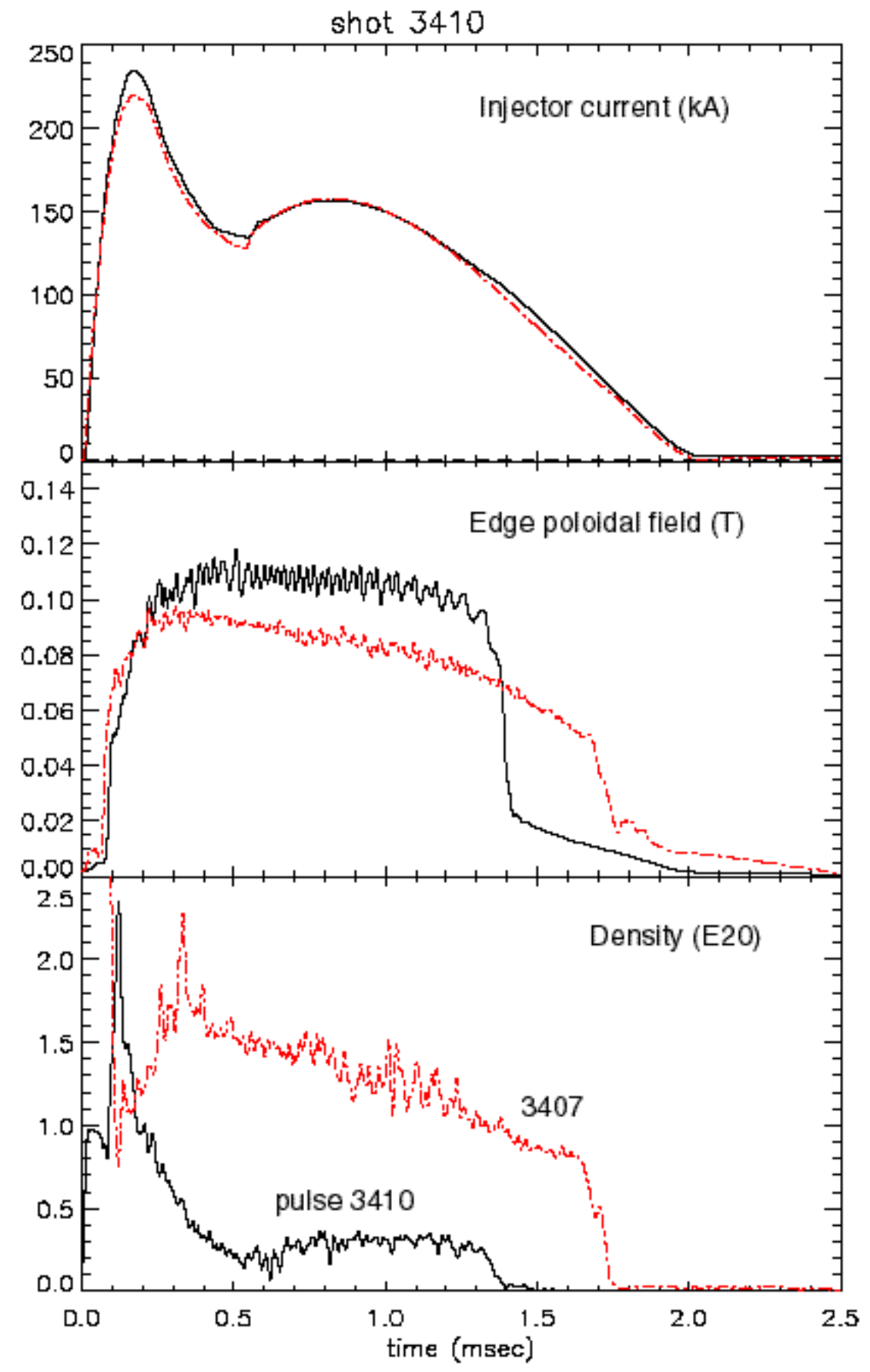

Figure 3. Sustained plasmas above (3407-dashed) and below (3410-solid) the threshold current. Top trace: injector current, middle: edge poloidal field, bottom: line-average plasma density. 


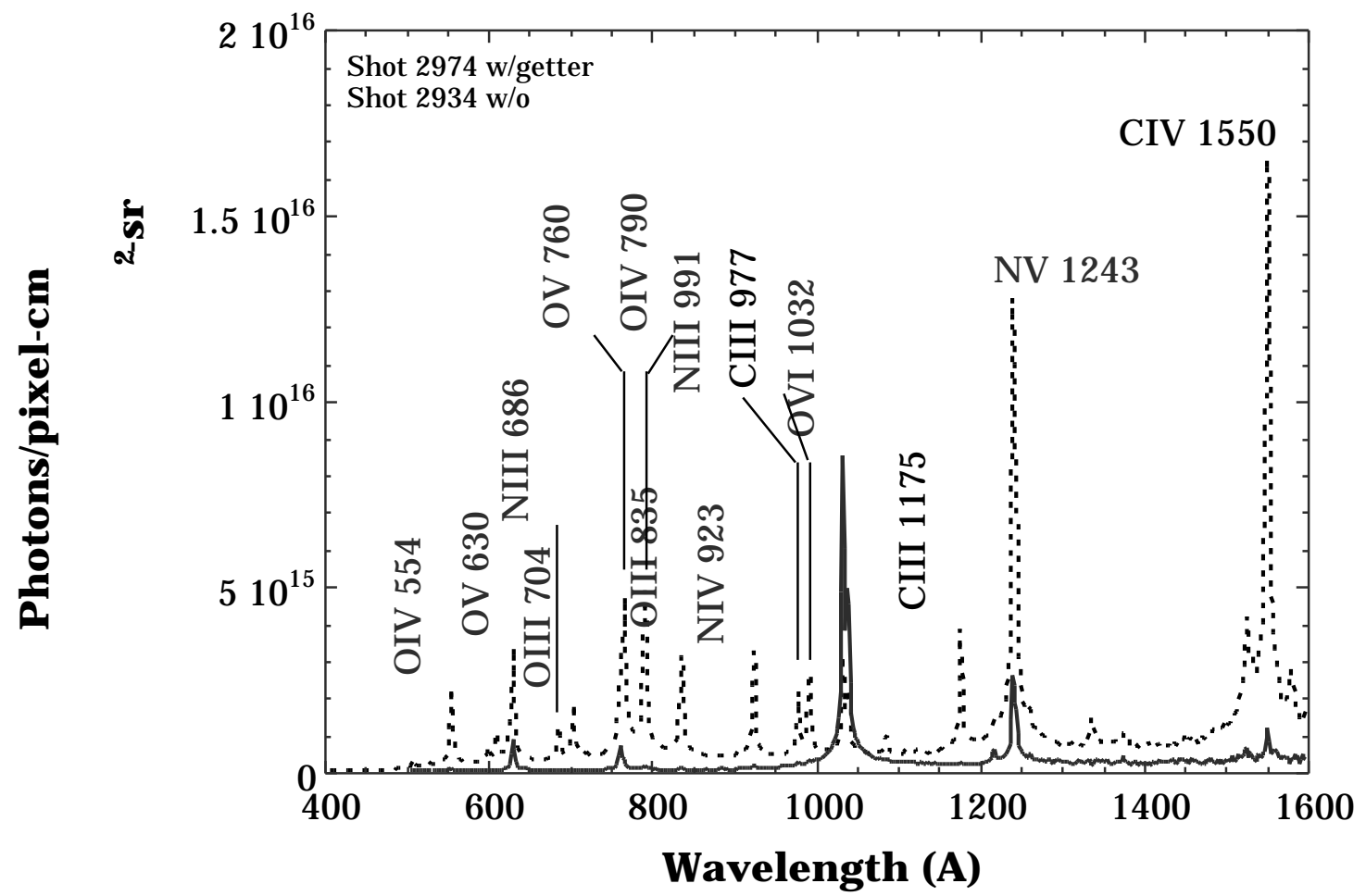

Figure 4. UV spectrum of a discharge without titanium gettering (dashed) and with gettering (solid). Li-like oxygen is the dominant radiator in discharges with gettering whereas before gettering the dominant radiators are low charge states of oxygen, Li-like carbon and Li-like nitrogen. Only low-Z impurities are observed on SSPX. 


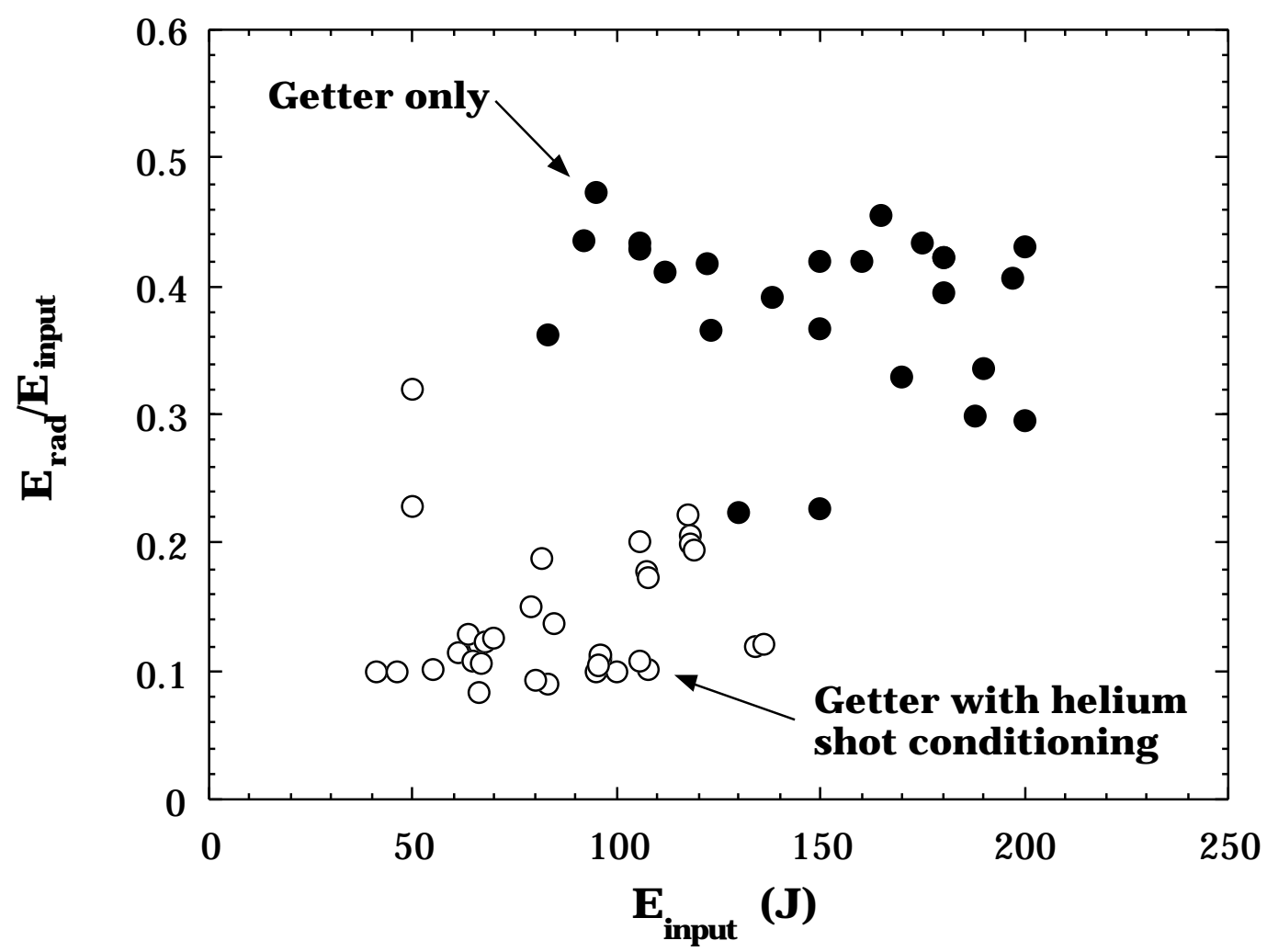

Figure 5. Fraction of total input energy radiated during discharges with titainium conditioning (solid circles) and with helium discharge and titainium gettering (open circles). $E_{\text {rad }}$ is measured with a thermistor that views a full radial cross section of the plasma at the midplane. Helium shot conditioning along with gettering decreases the total radiated energy as much as factor of 4. 


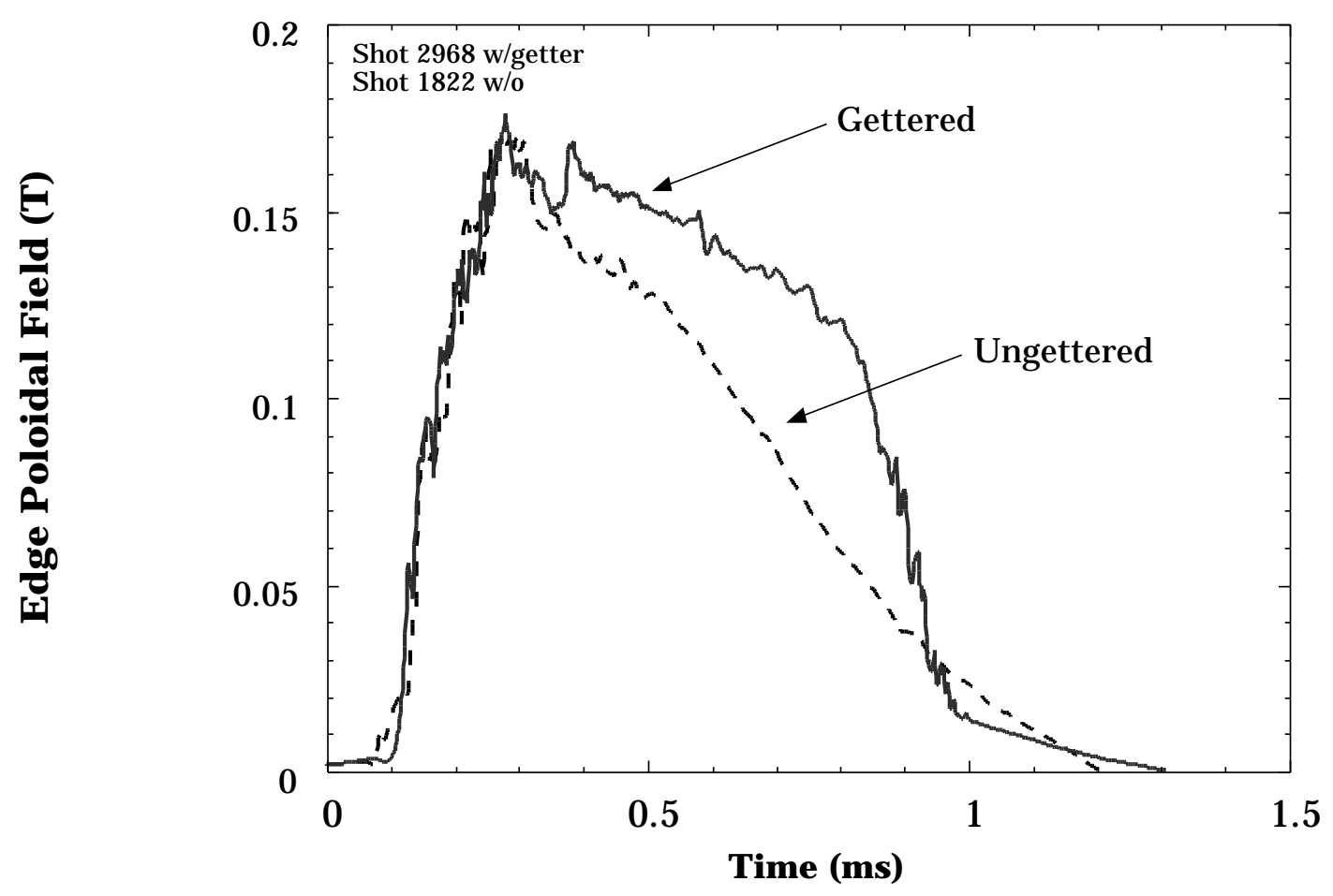

Figure 6. Edge poloidal field decay times increase a factor of two with gettering and helium shot conditioning. 